\title{
RESEÑA BIOGRÁFICA DEL DR. LUIS GUILLERMO BRENES SOBRADO
}
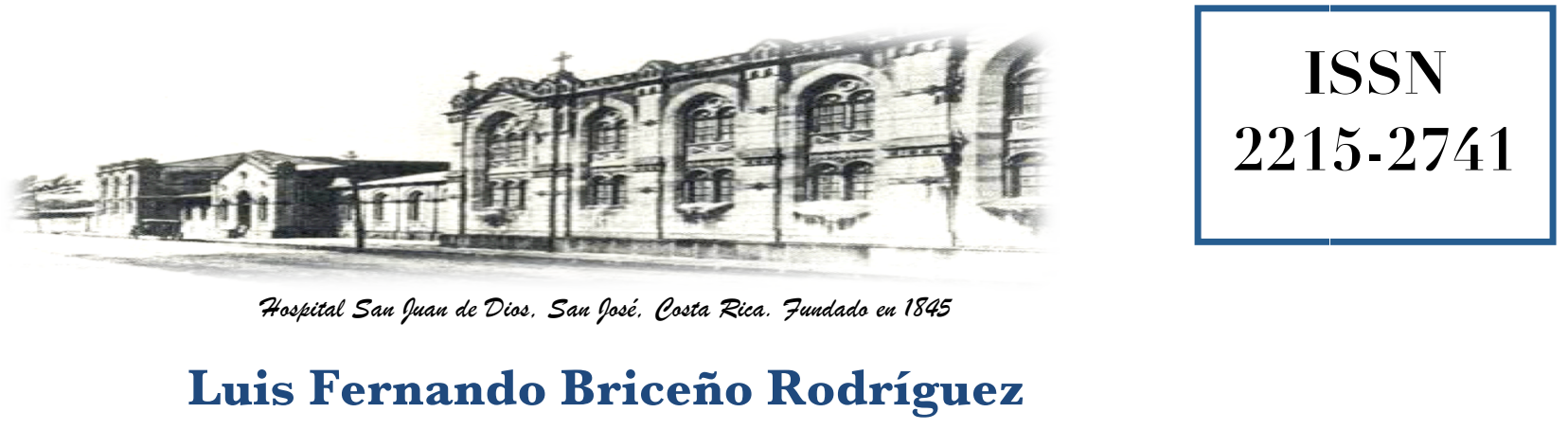

$\begin{array}{ll}\text { Recibido: } & 22 / 07 / 2013 \\ \text { Aceptado: } & 24 / 06 / 2013\end{array}$

HSJD

Luis Fernando Briceño Rodríguez ${ }^{1}$

\begin{abstract}
${ }^{1}$ Ex-Miembro Sección de Medicina - Hospital San Juan de Dios. Profesor Asociado Universidad de Costa Rica (pensionado). Correo electrónico: Ibriceno@racsa.co.cr
\end{abstract}

El doctor Luis Gmo. Brenes Sobrado fue Profesor Catedrático de la Escuela de Medicina de la Universidad de Costa Rica en el Hospital San Juan de Dios.

Nació en San José el 16 de febrero de 1928 y falleció el 25 de octubre del 2005, a los setenta y siete años de edad.

El doctor Brenes Sobrado fue un profesional de carácter firme y exigente, cuya formación académica le demandó una disciplina envidiable. Fue un científico con una calidad mental extraordinaria. Sus razonamientos y análisis siempre dejaban verdaderas enseñanzas.

Fue bachiller del Colegio Seminario en 1944. Bachiller de Ciencias en Química en Haverford College, Pensilvania - Estados Unidos de América (EUA) en 1949. Realizó estudios graduados en Bioquímica en Temple University, Pensilvania - EUA. Obtuvo el doctorado en
Medicina en la Escuela de Medicina de la Temple University, Pensilvania - EUA en 1954.

Realizó su Internado Rotatorio en el Hospital San Juan de Dios (HSJD) en 1954; y luego Residencia en Medicina Interna en el HSJD hasta 1957.

Realizó Internado en Medicina Interna en Temple University Hospital, EUA, en 1957.

Obtuvo el diploma del National Board of Medical Examiners de los EUA.

Realizó estudios graduados en Fisiología Renal y en Enfermedades Renales e Hipertensión en los departamentos de Fisiología y Medicina Interna en Temple University (EUA); y estudios en Fisiología Renal e Investigación en el Laboratorio de Riñón y Metabolismo Electrolítico en National Institute of Health (NIH) de los EUA, Bethesda en 1960, habiendo sido el único costarricense con ese privilegio. 


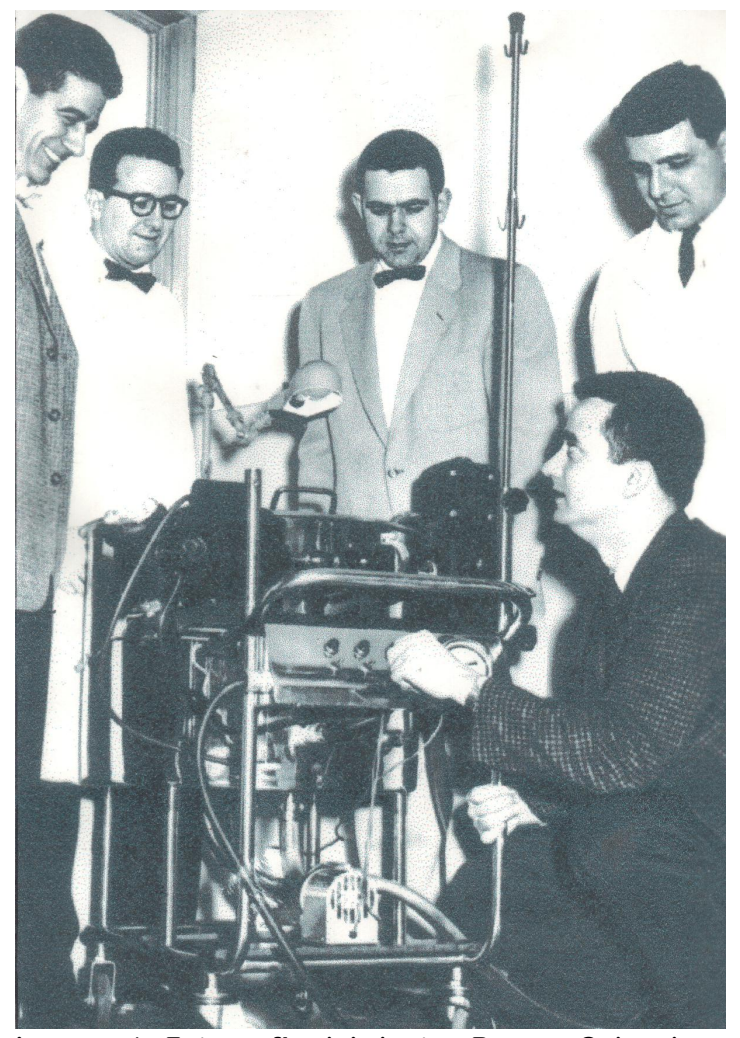

Imagen 1. Fotografía del doctor Brenes Sobrado, de cuclillas, frente al primer riñón artificial en la ciudad de Filadelfia - EUA, en compañía de renombrados médicos y científicos a nivel internacional, como Braunwald (autor de varios libros de Cardiología), y Nelson (autor del Tratado de Pediatría).

Concluyó la Residencia en Medicina Interna en Temple University Hospital (EUA) en 1962.

Se incorporó al Colegio de Médicos y Cirujanos de Costa Rica en 1956.

Además fue miembro de la American Federation for Clinical Research a partir de 1961; miembro de la Philadelphia Physiological Society a partir de 1960; y miembro del American College of Physicians desde 1969.

Poco después de regresar a nuestro país, inició como Médico Asistente Especialista en el Servicio de Medicina Interna No 2 del HSJD, impulsando y estimulando un carácter y una visión científica en el quehacer diario del Servicio; habiendo llegado a ser su Jefe de Servicio de 1965 a 1981.

Inició como profesor de Fisiología Renal en los albores de la Escuela de Medicina de la
Universidad de Costa Rica (UCR) a partir de 1963 y posteriormente profesor de Nefrología en los cursos de Medicina Interna de la UCR en el HSJD.

A solicitud del doctor Carlos Sáenz Herrera, fue consultor en Nefrología y Trastornos Hidroelectrolíticos y Ácido-Base en los inicios del Hospital Nacional de Niños, dedicando así varios años a la atención de la población infantil.

Creó el Servicio de Nefrología del HSJD, e inició el tratamiento de pacientes con insuficiencia renal aguda por medio de diálisis peritoneal. Con frecuencia nos platicaba sobre sus experiencias en la elaboración de las diferentes soluciones para la realización de los procedimientos dialíticos. Fue además promotor de las primeras biopsias renales en el HSJD.

Creó el Laboratorio de Nefrología e Investigación Clínica del HSJD, estando orgulloso de la participación del mismo en la realización de las investigaciones de los trabajos de graduación de los Residentes de Medicina Interna de los tres hospitales centrales: San Juan de Dios, México y Calderón Guardia; y se manifestaba optimista y satisfecho por el giro que en ese entonces tomaba la investigación clínica en este posgrado universitario. Siempre fue un luchador y un defensor de la Medicina Interna.

Fue Jefe de Sección de Medicina del HSJD de 1981 a 2004.

Publicó múltiples y excelentes artículos en reconocidas revistas científicas y médicas a nivel internacional, entre ellas: American Journal of Medicine, American Journal of Physiology, Clinical Research, Journal of the American Society of Nephrology, American Journal of Kidney Diseases y Kidney International.

Entre sus trabajos destacan la descripción por primera vez a nivel mundial de la "Acidosis Tubular Renal Proximal (ATRP) aislada hereditaria" y el estudio y seguimiento por más de treinta años de una familia con dicho trastorno en Costa Rica.

Sobresalen también sus estudios sobre glicerolisis en esferocitosis $y$ en coreaacantocitosis; estudios sobre los defectos en la excreción de amonio y los defectos en la 
excreción renal neta de ácido; los estudios de balanza ácido-base en acidosis tubular renal y los estudios sobre el efecto de verapamilo en la remisión de los hallazgos clínicos y de laboratorio en pacientes con corea-acantocitosis.

Su disciplina en el estudio y la calidad en la atención y en el seguimiento de sus pacientes eran extraordinarias.

Siempre se consideró sobre todo un verdadero Internista, apasionado del razonamiento integral de los casos clínicos, y apasionado de los fenómenos de membrana. Nunca separó el conocimiento y correlación existentes entre las ciencias básicas con la medicina clínica. Fue un hombre audaz, inteligente y brillante en la búsqueda de los "por qué".

Sus trabajos sobre Acidosis Tubular Renal Proximal han sido referencia en el Harrison's Principles of Internal Medicine (The Mc Graw Hill Companies, Inc.) y en importantes libros sobre trastornos hidroelectrolíticos y ácido-base como el Kokko \& Tannen - Fluids and Electrolytes (W.B. Saunders Company).

El doctor Brenes dedicó más de cuarenta años de su vida profesional a la Sección de Medicina del HSJD, a la consolidación científica del Laboratorio de Nefrología e Investigación Clínica y a la docencia. Fue pionero y entusiasta del estudio de los trastornos hidroelectrolíticos y ácido-base en nuestro país.

Sus trabajos científicos trascendieron nuestras fronteras y son un legado perdurable, que ha puesto muy en alto el nombre de la Medicina costarricense a nivel internacional.

Con su pensamiento y análisis de casos en diferentes reuniones de "sobremesa" (como él les llamó), nos fue ampliando el conocimiento crítico y analítico sobre la homeostasis del medio interno.

Nos enseñaba a "ensuciarnos las manos en investigación clínica", a "investigar haciendo". Nos inculcaba disciplina en la recolección de la información, en la realización de observaciones y en el análisis e interpretación de los datos. Le fascinaba el estudio y trabajo basado en el método científico; y sobre todo, fue muy enfático en la honestidad que debe existir en la investigación médica, dado a lo que consideraba problemas existentes en este campo.

A finales de 2005, las autoridades del HSJD y de la Sección de Medicina de ese entonces, aprobaron nombrar al Laboratorio de Nefrología e Investigación Clínica del hospital con su nombre (Imagen 2. Fotografía:).

A un gran "Maestro y Científico"; el sincero respeto y agradecimiento.

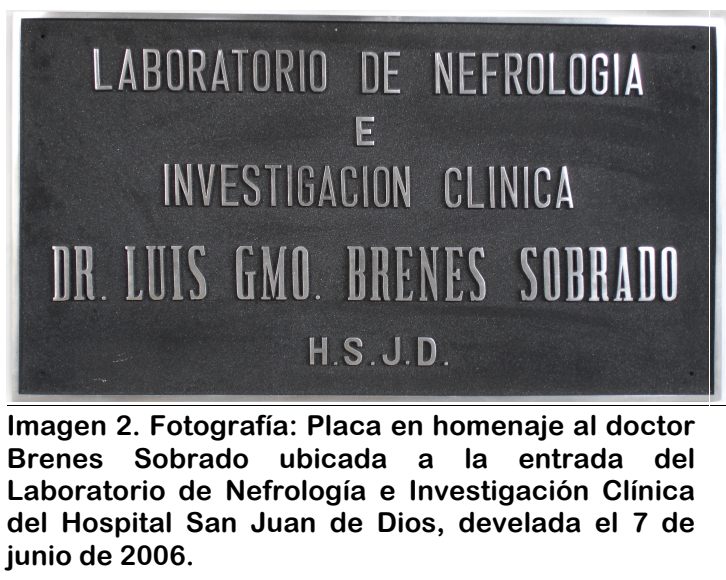

